\title{
On separation points of solutions to Prandtl boundary layer problem
}

\author{
By Shin'ya Matsui and Taira Shirota
}

(Received October 8, 1983)

\section{Introduction}

The equations for 2-dimensional stationary boundary layer theory of incompressible fluid past a rigid wall are

$$
\begin{aligned}
u u_{x}+v u_{y} & =\nu u_{y y}-p_{x}, \\
u_{x}+v_{y} & =0
\end{aligned}
$$

in the domain $D_{A}=\{(x, y) ; 0<x<A, 0<y<\infty\}$ (see [1], [8], [9], [10] and [11]). Here the subscripts $x$ and $y$ denote the partial differentiation with respect to the corresponding variable, $(x, y)$ are orthogonal coordinates in the boundary layer with $x$ representing the length along the wall and $y$ the perpendicular distance from the wall, $u=u(x, y)$ and $v=v(x, y)$ are the corresponding unknown velocity components. The constant $\nu$ is a viscous coefficient. Finally $p=p(x)$ is a pressure function. Let $U=U(x)$ be an exterior streaming speed; we assume that $p(x)$ and $U(x)$ satisfy the Bernoulli law and the origin $(0,0)$ is not a stagnation point, i.e.,

$$
\begin{gathered}
U(x) U_{x}(x)+p_{x}(x)=0, \\
U(0)>0 .
\end{gathered}
$$

The appropriate boundary conditions are

$$
\begin{aligned}
& u=v=0 \text { for } y=0 \text { and } u(x, y) \longrightarrow U(x) \text { as } y \rightarrow \infty, \\
& \text { uniformly in } x \text { on any compact subset of }[0, A) .
\end{aligned}
$$

In order to obtain a well-set problem, we suppose that at an initial position, say $x=0$, an initial datum $u_{0}(y)$ is assigned to the velocity component $u$, i. e.,

$$
u(0, y)=u_{0}(y) \quad(0 \leq y<\infty) .
$$

In this paper we study the existence of the separation point of the flow deterministically.

Hereafter, unless otherwise provided, we assume that the datum $u_{0}(y)$ belongs to $I^{2+\alpha}=I^{2+\alpha}(\nu, U)$ (for notations see Section 2) and that the speed $U(x)$ and the pressure gradient $p_{x}(x)$ have following properties: 
$U\left(X_{0}\right)=0$ for some point $X_{0}\left(0<X_{0}<\infty\right)$ and $U(x)>0$ for $0 \leq x<X_{0}$,

(1.6) The pressure $p(x)$ is sufficiently smooth, and if $p_{x}(c)=0$ at a point $x=c$, then a certain $N$-th derivative does not vanish at this point.

Now we mention our theorems.

THEOREM 1. For the problem (1.1), (1.3) and (1.4) there exists a solution $(u, v) \in P^{2}([0, s))$ such that the point $(s, 0)$ is its separation point and the inequality $0<s<X_{0}$ holds.

If we put $S\left(u_{0}\right)=s$ in Theorem 1 , then we obtain the mapping $S\left(u_{0}\right)$ from $I^{2+\alpha}$ to $\left(0, X_{0}\right)$.

THEOREM 2. (i) For the fixed viscosity, no separation point exists near the point $\left(X_{0}, 0\right)$ :

$$
\sup \left\{S\left(u_{0}\right) ; u_{0} \in I^{2+\alpha}\right\}<X_{0}
$$

(see [6]).

(ii) For the viscosity $\nu$ tending to zero, if the pressure gradient $p_{x}$ is monotone non increasing, there exist $u_{0}^{(\nu)} \in I^{2+\alpha}(\nu, U)$ and $u_{0} \in I^{2+\alpha}$ (without the compatibility condition) such that

$$
\begin{aligned}
& u^{(\nu)} \rightarrow u_{0} \quad \text { in } \quad B^{1}([0, \infty)) \quad \text { and } \\
& S\left(u_{0}^{(\nu)}\right) \rightarrow 0 \quad \text { as } \nu \rightarrow 0 .
\end{aligned}
$$

In physical or numerical experiments ([2], [4], [6], [12] and [13]) it is always assumed that the initial datum $u_{0}(y)$ is the constant $U(0)$ which does not belong to the class $I^{2+\alpha}$ and so that the separation point is independent of the viscosity $\nu$.

On the other hand, O. A. Oleinik [9] proved the local existence and the uniqueness of a solution $(u, v)$ in some domain $D_{A_{0}}$ to the problem (1.1), (1. 3) and (1.4) with a certain initial datum. Theorem 1 means that this local solution can be continued to the separation point. Recently Liu-Lee have tried to prove such a result. Their arguments do not appear plausible (their inequality (18. C). [7]]. To prove Theorem 1, we use essentially Lemma 1, 4 and 5 described below and to obtain (1.7) we compare our solution with the Blasius'.

In Section 2 we give a suitable definition of a separation point, notations mentioned above and the results on Oleinik's local solution with remarks about it. In Section 3 we prepare some lemmas and in Section 4 we prove Theorem 1 and 2. 


\section{Preliminaries}

For an interval $[a, A]$ let $B^{0}([a, A] \times[0, \infty))$ be the Banach space of uniformly bounded continuous functions defined over $[a, A] \times[0, \infty)$ with supermum norm. For $\alpha(0<\alpha \leq 2 / 3)$ and $y_{0}>0$ let $C^{\alpha}\left([a, A] \times\left[y_{0}, \infty\right)\right)$ be the set of continuous functions $u(x, y) \in C^{0}\left([a, A] \times\left[y_{0}, \infty\right)\right)$ which satisfy

$$
\left|u\left(x_{1}, y_{1}\right)-u\left(x_{2}, y_{2}\right)\right| \leq M\left\{\left|x_{1}-x_{2}\right|^{1 / 2}+\left|y_{1}-y_{2}\right|\right\}^{\alpha}
$$

for $\left(x_{i}, y_{i}\right) \in[a, A] \times\left[y_{0}, \infty\right)(i=1,2)$ and $M=M\left(y_{0}, u\right)$, and let $C^{\alpha}([a, A] \times$ $(0, \infty))=\bigcap_{y_{0}>0} C^{\alpha}\left([a, A] \times\left[y_{0}, \infty\right)\right)$. Furthermore let $B^{\alpha}([a, A] \times(0, \infty))=B^{0}([a, A]$ $\times[0, \infty)) \cap C^{\alpha}([a, A] \times(0, \infty))$. We also define $C^{\alpha}((0, \infty)), B^{0}((0, \infty))$ and $B^{2+\alpha}((0, \infty))$ by the analogous way.

Then we define the space of the initial data:

$$
\begin{aligned}
& I^{2+\alpha}(\nu, U)=\left\{u(y) \in C^{2}([0, \infty)) \cap B^{2+\alpha}((0, \infty)) ; u(0)=0,\right. \\
& u_{y}(0)>0, u_{y}(y) \geq 0 \text { for } y \geq 0, u \rightarrow U(0) \text { as } y \rightarrow \infty \text { and } \\
& \left.\nu u_{y y}(y)-p_{x}(0)=O\left(y^{2}\right) \text { as } y \rightarrow 0\right\} .
\end{aligned}
$$

The condition in $I^{2+\alpha}$

$$
\nu u_{y y}(y)-p_{x}(0)=O\left(y^{2}\right) \text { as } y \rightarrow 0
$$

is a strong compatibility condition.

The space of the solutions to the problem (1.1), (1.3) and (1.4) is given as follows :

Let $P^{2}([a, A])$ be a set of all functions $(u, v)$ such that

(i) $u, u_{x}, u_{y}, u_{y y}, v$ and $v_{y} \in C^{0}([a, A] \times[0, \infty))$,

(ii) $u(x, y)>0$ in $[a, A] \times[0, \infty)$,

(iii) $u_{y}(x, 0)>0$ for $x \in[a, A]$,

(iv) $u, u_{y}, u_{y y} \in B^{0}([a, A] \times[0, \infty))$.

Then we define the space by

$$
P^{2}([0, A))=\bigcap_{0<A^{\prime}<A} P^{2}\left(\left[0, A^{\prime}\right]\right) .
$$

Now we define the separation point of a solution to the problem (1.1), (1.3) and (1.4):

Definition. A point $(s, 0)$ is a separation one of a solution $(u, v)$ to our problem in the domain $D_{s}$, if the solution $(u, v)$ belongs to $P^{2}([0, s))$ and for some sequence $\left(x_{n}, y_{n}\right)$ in $[0, s) \times[0, \infty)$

$$
\left(x_{n}, y_{n}\right) \rightarrow(s, 0) \text { and } u_{y}\left(x_{n}, y_{n}\right) \rightarrow 0 \text { as } n \rightarrow \infty \text {. }
$$


Here we note that physicists call $(s, 0)$ a separation point if $u_{y}(s, 0)=0$ for a solution $(u, v)$ (see [4], [12]). Our definition of the separation point is the same one as the above for a solution $(u, v) \in P^{2}([0, s))$ with $u, u_{y} \in$ $C^{0}([0, s] \times[0, \infty))$. But to our knowledge, the extstence of such an exact solution is not yet proved under our assumptions.

We consider the transformation of the independ variables in the system (1.1) of the form

$$
x=x, \quad \phi=\phi(x, y),
$$

where

$$
u=\phi_{y}(x, y), \quad v=-\phi_{x}(x, y), \quad \phi(x, 0)=0 .
$$

If we put $w(x, \phi)=u^{2}(x, y)$, then the transformation (2.2) reduces the problem (1.1), (1.3) and (1.4) to the Von Mises' form:

$$
L(w)=\nu \sqrt{w} w_{\psi \psi}-w_{x}=2 p_{x} \text { in } G_{A}
$$

with the conditions

$$
\begin{aligned}
& w(x, 0)=0, w(x, \phi) \rightarrow U^{2}(x) \text { as } \phi \rightarrow \infty \\
& \text { uniformly in } x \text { on }[0, A), \\
& w(0, \phi)=w_{0}(\phi)
\end{aligned}
$$

where

$$
\begin{aligned}
G_{\boldsymbol{A}}= & \{(x, \phi) ; 0<x<A, 0<\phi<\infty\}, \\
& w_{0}\left(\int_{0}^{y} u_{0}(t) d t\right)=u_{0}{ }^{2}(y) .
\end{aligned}
$$

Let $I_{M}^{2+\alpha}=\left\{w(\phi) ; w\left(\int_{0}^{y} u(t) d t\right)=u^{2}(y), u \in I^{2+\alpha}\right\}$.

Then $w(\phi) \in I_{M}{ }^{2+\alpha}$ if and only if

$$
\begin{aligned}
w, w_{\phi}, \sqrt{w} w_{\psi \phi} \in B^{0}([0, \infty)) \cap C^{\alpha}((0, \infty)), \\
w(0)=0, w_{\phi}(0)>0, w_{\psi}(\phi) \geq 0 \text { for } \phi \geq 0, \\
w(\phi) \rightarrow U^{2}(0) \text { as } \phi \rightarrow \infty \text { and } \\
\mu(\phi)=\nu \sqrt{w(\phi)} w_{\psi \psi}(\phi)-2 p_{x}(0)=0(\phi) \text { as } \phi \rightarrow 0 .
\end{aligned}
$$

Let $P_{M^{2+\alpha}}([0, A])=\{w(x, \phi)$;

$$
\begin{aligned}
& w, \quad w_{x} \quad w_{\psi}, \sqrt{w} w_{\psi \psi} \in B^{0}\left(\bar{G}_{A}\right) \cap C^{\alpha}([0, A] \times(0, \infty)), \\
& \left|w_{x}\right| \leq K \phi^{1-\beta} \text { and } w_{\psi} \geq m \text { in }[0, A] \times\left[0, \phi_{1}\right] \text { and } \\
& \left.w(x, \phi)>l \text { for } \phi>\phi_{1}\right\},
\end{aligned}
$$


where positive constants $\phi_{1}, m, l$ depend on $w$. Furthermore for any $\beta$ $(0<\beta<1 / 2)$ the positive constant $K$ depends on $\phi_{1}, \beta$ and $w$.

$$
\text { Let } P_{M^{2+\alpha}}([0, A))=\bigcap_{0<A^{\prime}<A} P_{M^{2+\alpha}}\left(\left[0, A^{\prime}\right]\right) \text {. }
$$

Here we summarize the results and remarks on Oleinik's local solutions (see [9] or [10]) :

(I) A solution $(u, v) \in P^{2}([0, A])$ of the problem (1.1), (1.3) and with the initial datum $u_{0} \in I^{2+\alpha}$ (without $u_{0_{y}}(y) \geq 0$ for $y>0$ ) exists, if a solution $w \in$ $P_{M}{ }^{2+\alpha}([0, A])$ of the problem (2.3), (2.4) and (2.5) exists.

(II) Oleinik assumes that the initial datum $u_{0}$ belongs to $B^{2+\alpha}([0, \infty))$, but our initial datum $u_{0}$ belongs to $C^{2}([0, \infty)) \cap B^{2+\alpha}((0, \infty))$ and this assumption of regularity is enough for the existence of Oleinik's local solution.

Here we note that $I_{M^{2+\alpha}}$ contains a sufficiently large number of elements. In fact let $w_{0}(\psi)$ be $\left\{m_{0} \psi+B_{1} \psi^{3 / 2}-B_{2} \psi^{2}\right\} \chi(\phi)+(1-\chi(\psi)) U^{2}(0)$, where $m_{0}>0$, $B_{1}=8 p_{x}(0) / 3 \nu \sqrt{m_{0}}, B_{2}=4 p_{x}{ }^{2}(0) / 3 \nu^{2} m_{0}{ }^{2}$ and $\chi(\phi)$ is a function $\in C^{\infty}([0, \infty))$ such that $\chi_{\dot{\phi}} \leq 0,0 \leq \chi \leq 1, \chi=1$ for $\phi \ll 1$, and $\sup (\operatorname{supp} \chi) \ll 1$. Then it is easy to see that $w_{0}$ belongs to $I_{M}{ }^{2+\alpha}$.

(III) For small $\varepsilon>0$, let $G_{A}^{\varepsilon}=\{(x, \phi) ; 0<x<A, 0<\phi<1 / \varepsilon\}$. Then for some positive constant $A_{0}$ and any sufficiently small $\varepsilon$, there exists the approximate positive solution $w_{c}(x, \phi)$ defined on $G_{A_{0}}^{s}$, which satisfies $(2.3)$ with boundary conditions :

$$
\begin{aligned}
& w_{\varepsilon}(0, \phi)=w_{0}(\varepsilon+\phi), w_{\bullet}(x, 0)=w_{0}(\varepsilon) \exp \left\{\mu(\varepsilon) x / w_{0}(\varepsilon)\right\}, \\
& w_{\bullet}(x, 1 / \varepsilon)=w_{0}(\varepsilon+1 / \varepsilon) \exp \left\{\mu(\varepsilon+1 / \varepsilon) x / w_{0}(\varepsilon+1 / \varepsilon)\right\} .
\end{aligned}
$$

Then the solution $w_{6}(x, \phi)$ belongs to $P_{M^{2}}{ }^{2+\alpha}\left(\overline{G_{A_{0}}}\right)$, where the last functional space is defined as in $\mathrm{P}_{M^{2+\alpha}}([0, A])$, but we must replace $B^{0}\left(\bar{G}_{A_{0}}\right)$ and $C^{\alpha}\left(\left[0, A_{0}\right]\right.$ $\times(0, \infty))$ by $B_{0}\left(\overline{G_{A_{0}}^{c}}\right)$ and $C^{\alpha}\left(\left[0, A_{0}\right] \times(0,1 / \varepsilon]\right)$ respectively.

Here we must mention that the solution $w_{c}(x, \phi)$ has the following properties :

All constants in $P_{M^{2+\alpha}}$ (i. e., $\left.\alpha, m, \phi_{1}, K, l\right)$ and supremum

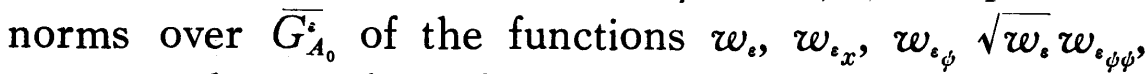
$w_{\varepsilon}^{\beta-1} w_{\epsilon_{x}}$ do not depend on $\varepsilon$.

Finally the unique local solution $w \in P_{M^{2+\alpha}}\left(\left[0, A_{0}\right]\right)$ is obtained as follows : For a subsequence $\left\{w_{\varepsilon}\right\}$ and any $N \gg 1$

$$
w_{\varsigma^{\prime}} \rightarrow w \quad \text { as } \quad \varepsilon^{\prime} \rightarrow 0 \quad \text { in } \quad C^{0}\left(\left[0, A_{0}\right] \times[0, N]\right)
$$

and $w_{\iota^{\prime} x}, w_{\epsilon^{\prime} \psi^{\prime}}, \sqrt{w_{\iota^{\prime}}} w_{\epsilon^{\prime}{ }_{\psi}{ }^{\prime}}$ converge the corresponding functions with respect 
to $w$ as $\varepsilon^{\prime} \rightarrow 0$ in $C^{0}\left(\left[0, A_{0}\right] \times[1 / N, N]\right)$, provided $\alpha \leq 2 / 3$. Here we note that $w(x, \phi) \rightarrow U^{2}(x)$ as $\phi \rightarrow \infty$ uniformly in $x$ on $\left[0, A_{0}\right]$.

Now by the transformation (2.2), we have $u_{y}=\frac{k}{2} w_{\psi}$. Therefore we may define the separation point of the solution $w$ to the problem (2.3), (2.4) and $(2.5)$ by the same way as above; i.e., a point $(s, 0)$ is the separation one of a solution $w(x, \psi)$ to our problem, if the solution $w(x, \phi)$ belongs to $P_{M^{2+\alpha}}([0, s))$ and for some sequence $\left(x_{n}, \psi_{n}\right)$ in $[0, s) \times[0, \infty)$

$$
\left(x_{n}, \phi_{n}\right) \rightarrow(s, 0) \quad \text { and } \quad w_{\psi}\left(x_{n}, \phi_{n}\right) \rightarrow 0 \quad \text { as } n \rightarrow \infty .
$$

We also denote this separation point by $S\left(w_{0}\right)$. Then, in fact, we obtain that $S\left(u_{0}\right)=S\left(w_{0}\right)$ for $u_{0}$ corresponding to $w_{0} \in I_{M}{ }^{2+\alpha}$ by $(2.2)$.

\section{Lemmas}

Lemma 1. Let $w(x, \phi)$ be Oleinik's local solution. Then there exist positive constants $M_{1}$ and $\lambda$ such that

$$
\left|w_{x}\right| \leq M_{1} \phi \text { for } 0 \leq x \leq A_{0} \text { and } 0 \leq \phi \leq \lambda .
$$

Proof. Let $w_{\varepsilon}(x, \phi)$ be the approximate solution given in Section 2.

We put $E=\left\{(x, \phi) ; 0 \leq x \leq A_{0}, 0 \leq \phi \leq \lambda\right\}$, where the small positive constant $\lambda$ will be determined latter. From the fact (III), if we show

$$
\left|w_{\varepsilon}^{-1} w_{\varepsilon_{x}}\right| \leq M_{1} \quad \text { in } E
$$

for some $M_{1}, \lambda$ and sufficiently small $\varepsilon>0$, then we obtain (3.1). Hence it is sufficient to show the above inequality.

Since $w_{\diamond}>0$ (see (III)), we may put $g_{\imath}(x, \psi)=\ln \left(w_{\iota}(x, \psi)\right)$, then from $(2.3)$ $g_{c}$ satisfies the following equation :

$$
\nu g_{c_{\phi \psi} \psi} e^{3 g_{\iota} / 2}+\nu\left(g_{\iota_{\psi}}\right)^{2} e^{3 g_{\iota} / 2}-e^{g_{\star}} g_{\iota_{x}}=2 p_{x} .
$$

Differentiating (3.2) with respect to $x$, we obtain an equation for $g_{\iota_{x}}=h_{\mathfrak{c}}$ :

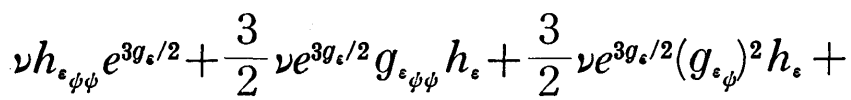

$$
\begin{aligned}
& 2 \nu e^{3 g_{\mathrm{s}} / 2} g_{\varepsilon_{\psi}} h_{\varepsilon_{\psi}}-e^{g_{\mathrm{c}}}\left(h_{\varepsilon}\right)^{2}-e^{g_{\mathrm{s}}} h_{\varepsilon_{x}}=2 p^{\prime \prime} .
\end{aligned}
$$

Replacing $\nu g_{\iota_{\phi \psi}} \exp 3 g_{\iota} / 2$ by its value from (3.2), we can write the equation for $h_{\varepsilon}$ in the form:

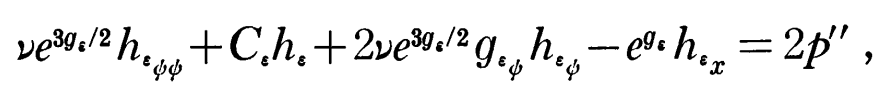

where 


$$
C_{\iota}=\frac{1}{2} h_{\imath} e^{g_{\iota}}+3 p^{\prime}=\frac{1}{2} w_{\iota_{x}}+3 p^{\prime} .
$$

From (2.8), there exists a positive constant $M_{2}$ independent of $\varepsilon$ such that

$$
\left|C_{\varepsilon}\right| \leq M_{2} \quad \text { in } \overline{G_{A_{0}}} .
$$

Therefore (3.3) implies that $j_{\varepsilon}=h_{\varepsilon} \exp \left\{-\left(M_{2}+1\right) x\right\}$ satisfies

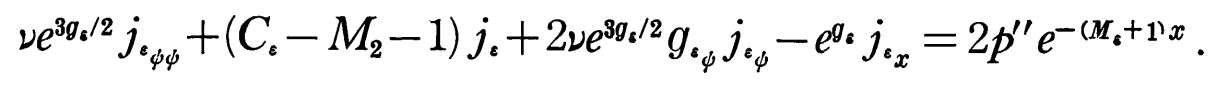

Now if $j_{\text {。 }}$ attains its maximum in $E$ without the lines $x=0, \phi=0$ and $\phi=\lambda$, then at this point we have

$$
\nu e^{3 g_{\mathrm{s}} / 2} j_{\iota_{\varphi \psi}}-e^{y_{\mathrm{s}}} j_{\iota_{x}} \leq 0 \text { and } j_{\iota_{\varphi}}=0 .
$$

Hence from (3.4) and (3.5) we deduce

$$
j_{\varepsilon} \leq \frac{2 p^{\prime \prime} \exp \left\{-\left(M_{2}+1\right) x\right\}}{C_{c}-M_{2}-1} \leq M_{3} \text { at the maximum point, }
$$

where the positive constant $M_{3}$ does not depend on $\varepsilon>0$.

By the same argument, at the minimum point of $j_{c}$ in the same domain of $E$ we have

$$
j_{6}>-M_{3} \text {. }
$$

If $\varepsilon$ and $\lambda$ are sufficiently small, from (2.6) it follows that $h_{\varepsilon}(0, \phi)=$ $w_{0}(\varepsilon+\phi)^{-1} \mu(\varepsilon+\phi)=O(1)$ and $h_{\varepsilon}(x, 0)=w_{0}(\varepsilon)^{-1} \mu(\varepsilon)=O(1)$. Furthermore the fact (III) in Section 2 implies $h_{c}(x, \phi)=O(1)$.

Thus from (3.6) and (3.7) we conclude that

$$
\left|h_{\varepsilon}\right| \leq M_{4} \text { in } E,
$$

where the positive constant $M_{4}$ does not depend on $\varepsilon$. This proves Lemma 1.

q. e. d.

It is easy to see that (2.3) and (3.1) imply the following Corollary.

Corollary. Let $w(x, \phi)$ be as in Lemma 1. Then the section $w(x, \cdot)$ satisfies the compatibility condition (2.6) for any $x\left(0 \leq x \leq A_{0}\right)$.

Lemma 2. ([8]). Let $w(x, \phi)$ be a solution to the problem (2.3), (2.4) and (2.5) belonging to $P_{M}{ }^{2+\alpha}([0, A])$. Then $w(x, \phi)$ is monotone nondecreasing with respect to $\phi$ in $\bar{G}_{A}$.

Proof. We use the methods of the proof in [5] here.

Since $w(x, \phi)$ belongs to $P_{M}{ }^{2+\alpha}$, for sufficiently small $\delta>0$ there exist positive constants $m$ and $M$ such that

$$
m \leq w \leq M \text { in } \bar{G}_{\boldsymbol{A}} \cap\{\phi \geq \delta\},
$$




$$
\begin{aligned}
& \left|w_{\psi}\right| \leq M \text { in } \bar{G}_{\boldsymbol{A}} \text { and } \\
& w_{\psi} \geq m \text { in } \bar{G}_{\boldsymbol{A}} \cap\{0 \leq \psi \leq \delta\} .
\end{aligned}
$$

Differentiating the equation (2.3) with respect to $\phi$, we obtain an equation for $z=w_{\varphi}$ :

$$
P(z)=\nu \sqrt{w} z_{\psi \psi}+\frac{\nu}{2} w^{-1 / 2} w_{\psi} z_{\psi}-z_{x}=0 .
$$

We put $g(x, \phi)=R^{-2} M\left(\psi^{2}+K x\right) e^{K x}+z(x, \phi)$ and $E_{r, R}=\{(x, \phi) ; 0 \leq x \leq A, r \leq$ $\phi \leq R\}$ for $R \gg r>0$, where a positive constant $K$ will be determined latter.

From (3.11) we obtain an equation for $g$ :

$$
P(g)=R^{-2} M e^{K x}\left(2 \nu \sqrt{w}+\nu w^{-1 / 2} w_{\psi} \psi-K-K \psi^{2}-K^{2} x\right) .
$$

If $K>\nu\left(2 M^{1 / 2}+m^{-1 / 2} M\right)$, taking account of (3.8), (3.9) and (3.10), we have

$$
P(g) \leq R^{-2} M e^{K x}\left(2 \nu M^{1 / 2} \psi^{2}+\nu m^{-1 / 2} M \psi^{2}-K \psi^{2}\right)<0 \text { in } E_{1, R}
$$

and

$$
P(g) \leq R^{-2} M e^{K x}\left(2 \nu M^{1 / 2}+\nu m^{-1 / 2} M-K\right)<0 \text { in } E_{\delta, 1} .
$$

Hence we see $P(g)<0$ in $E_{\delta, R}$. Furthermore on the lines $x=0, \phi=\delta$ and $\phi=R$ we have the following: Since $w_{0} \in I_{M}^{2+\alpha}$ and $w_{\phi}=2 u_{y}, g(0, \phi)=$ $R^{-2} M \psi^{2}+z(0, \phi)=R^{-2} M \psi^{2}+w_{0_{\psi}}(\psi) \geq 0$ and (3.10) implies that $g(x, \boldsymbol{\delta}) \geq \boldsymbol{z}(x, \boldsymbol{\delta})$ $=w_{\psi}(x, \delta) \geq 0$. Finally $g(x, R) \geq M+z(x, R)=M+w_{\psi}(x, R) \geq 0$ by (3.9).

Therefore by the maximum principle we obtain $g \geq 0$ in $E_{\delta, R}$. Then if $R \rightarrow \infty$, we have $w_{\phi}(x, \phi) \geq 0$ for $\phi \geq \delta$. Taking account of (3.10), we conclude that our assertion for $\phi<\delta$ is valid.

q. e. d.

Lemma 3. There exists a point $x_{0}>0$ such that the pressure gradient $p_{x}(x)$ is monotone decreasing in $\left[x_{0}, X_{0}\right]$.

Proof. From (1.5) and the Bernoulli law (1.2) we have $p_{x}\left(X_{0}\right)=0$. Taking account of (1.6), we have that for some constant $a \neq 0$ and $x$ near $X_{0}$

$$
p_{x}(x)=a\left(X_{0}-x\right)^{N}+O\left(\left(X_{0}-x\right)^{N+1}\right) .
$$

Now if the constant $a$ is negative, then $p_{x}(x)<0$ near $X_{0}$. Therefore an inequality $U^{2}(x)=2\left(p\left(X_{0}\right)-p(x)\right)<0$ holds for such $x$, since (1.2) and (1.5) are assumed. This contradiction means that the pressure gradient $p_{x}$ is nonnegative and monotone decreasing on some interval $\left[x_{0}, X_{0}\right]$. q.e.d.

Lemma 4. Suppose that the pressure gradient $p_{x}$ is monotone non increasing on $[0,1]$ and the point $X_{0}$ in (1.5) equlas to 1 . Then for any positive constant $k$ there exist positive constants $\gamma$ and $A<1$ such that if 
$w(x, \phi)$ is a solution to the problem (2.3), (2.4) and (2.5) belonging to $P_{M}^{2+\alpha}([0, A)) \cap C^{0}([0, A] \times[0, \infty))$ and if

$$
w_{0}(\psi) \leq k \psi \int_{0}^{1} p_{x}(t) d t \quad \text { for } \quad 0 \leq \psi \leq 2 / k,
$$

then

$$
\begin{aligned}
\omega(x, \phi) & \leq F(x, \phi) \\
& \equiv k \psi\left\{2 \int_{x / A}^{1} p_{x}(t) d t(1-\gamma \psi)+2 \gamma \psi \int_{x}^{1} p_{x}(t) d t\right\}
\end{aligned}
$$

for $0 \leq x \leq A, 0 \leq \psi \leq 1 / \gamma$.

Proof. Since the initial datum $w_{0} \in I_{M^{2+\alpha}}$, we may find a positive constant $k$ such that (3.13) is valid.

Now the above inequality implies that

$$
w_{0}(\psi) \leq 2\left(k \psi-k^{2} \psi^{2} / 4\right) \int_{0}^{1} p_{x}(t) d t \text { for } 0 \leq \psi \leq 2 / k .
$$

Furthermore for an arbitrary constant $A<1$ we have

$$
w(x, \phi) \leq 2 \int_{x}^{1} p_{x}(t) d t \quad \text { in }[0, A] \times[0, \infty),
$$

if a solution $w$ is well defined in $[0, A] \times[0, \infty)$ as above. In fact, (1.2) and (1. 5) imply that $w(0, \phi)=w_{0}(\psi) \leq U^{2}(0)=U^{2}(1)+2 p(1)-2 p(0)=2 \int_{0}^{1} p_{x}(t) d t$ and $w(x, 0)=0 \leq 2 \int_{x}^{1} p_{x}(t) d t$ and $L(w)-L\left(2 \int_{x}^{1} P_{x} d t\right)=0$. Hence by the maximum principle (3.16) holds (see the proof of Theorem 3 in [9]).

Moreover we define $H(x, \phi)$ as follows:

$$
H(x, \psi)=2\left(k \psi-k^{2} \psi^{2} / 4\right) \int_{x}^{1} p_{x}(t) d t \text { for } 0 \leq \psi \leq 2 / k .
$$

Then it follows from (3.15) and (3.16) that an inequality $w(x, \phi) \leq H(x, \phi)$ holds on the lines $x=0, \phi=0$ and $\phi=2 / k$. Furthermore by simple calculation we obtain

$$
L(H)-L(w) \leq 0 \quad \text { in } \quad[0, A) \times[0,2 / k]
$$

for any $A<1$.

Hence by the maximum principle we have

$$
w(x, \phi) \leq H(x, \phi) \quad \text { in } \quad[0, A] \times[0,2 / k] .
$$

Now we prove the inequality (3.14) for some $\gamma$ and $A<1$. From (3.13) and (3.17), the inequality (3.14) holds on the lines $x=0, \phi=0$ and $\phi=1 / \gamma$ 
for $2 \gamma>k$. Furthermore, from the inequalities $A<1, k<2 \gamma$, the monotoneity of $p_{x}$ and (1.5), we deduce the following:

$$
\begin{aligned}
L(F) & =\nu \sqrt{F} F_{\psi \psi}-F_{x} \\
& \leq 4 \nu k \gamma p_{x}(x)(x / A-x)\left(2 k \gamma^{-1} \int_{0}^{1} p_{x}(t) d t\right)^{1 / 2}+p_{x}(x) \frac{2 k}{A} \psi \\
& \leq 2 c k^{3 / 2} \gamma^{1 / 2}(1-A) p_{x}(x)+2 k p_{x} / \gamma A, \\
& \text { for } 0 \leq \psi \leq 1 / \gamma, 0 \leq x \leq A,
\end{aligned}
$$

where $c^{2}=8 \nu^{2} \int_{0}^{1} p_{x}(t) d t$.

Hence an inequality $L(F) \leq 2 p_{x}(x)$ holds for $0 \leq x \leq A$ and $0 \leq \psi \leq 1 / \gamma$ if an inequality

$$
c k^{3 / 2} \gamma^{1 / 2}(1-A) A+k / \gamma \leq A
$$

holds.

Therefore considering the quadratic inequality in $A$, we may find $A<1$ for $\gamma>k$. Then from (3.18) it follows that $L(F)-L(w) \leq 0$ for $0 \leq x<A$ and $0 \leq \phi \leq 1 / \gamma$. Thus by the maximum principle we have the inequality (3. 14)

q. e. d.

We note that the above quadtatic inequality is valid for

$$
\gamma=\left(1+D^{1 / 2}\right)^{2} / c_{1} k^{3} \text { and } A=1-2 /\left(1+D^{1 / 2}\right),
$$

where $D=1+c_{2} k^{4}$, provided $c_{1}=3^{2} c^{2}$ and $c_{2}=3^{3} c^{2}$. Furtheremore the argument described below and (3.19) imply that for the separation point $(s, 0)$

$$
0<s \leq A=1-2 /\left(1+D^{1 / 2}\right) .
$$

COROllary. There exists no solution w $(x, \psi)$ to the problem (2.3), (2.4) and (2.5) in $G_{X_{0}}$, which belongs to the class $P_{M}{ }^{2+\alpha}\left(\left[0, X_{0}\right)\right)$ and rohose section $w(x, \cdot)$ belongs to $I_{M}{ }^{2+\alpha}$ for any $x\left(0 \leq x<X_{0}\right)$.

Proof. Assume that the above corollary is not valid. Let $x_{0}$ be the point in Lemma 3. Then, from our assumption, we may consider the line $x=x_{0}$ as the initial position and the section $w\left(x_{0}, \cdot\right)$ as the initial datum. Therefore by a coordinates transformation, setting $x_{0}=0$ and $X_{0}-x_{0}=1$, we may consider that the solution $w(x, \phi)$ satisfies the assumptions in Lemma 4 . Then from $w(x, 0)=0$ and Lemma 4 , we have

$$
\frac{w(x, \phi)-w(x, 0)}{\psi} \leq 2 k \int_{x / A}^{1} p_{x}(t) d t(1-\gamma \psi)+2 \gamma k \psi \int_{x}^{1} p_{x}(t) d t
$$

for $0 \leq x \leq A$ and $0 \leq \psi \ll 1$.

Therefore if $\phi \downarrow 0$ and $x \uparrow A$, then we obtain 


$$
w_{\psi}(A, 0) \leq 0 .
$$

This proves the corollary.

q. e. $d$.

For a point $X_{1}\left(0<X_{1}<X_{0}\right)$, let $k_{1}$ and $k_{2}$ be $\min _{0 \leq x \leq X_{1}} U^{2}(x)$ and $\max _{0 \leq x \leq X_{1}}\left|2 p_{x}(x)\right|$ respectively. Furthermore let $W$ be the subset of $I_{M}{ }^{2+\alpha}$ such that for $w_{0}(\phi)$ $\in W$

$$
\inf \left\{w_{0_{\psi}}(\psi) ; 0 \leq \psi \leq \psi_{0}\right\} \geq k
$$

where $\psi_{0}$ and $k$ are, $a$ priori, sufficiently small positive numbers.

Then we have

Lemma 5. For any $w_{0} \in W$, the constant $A_{0}$ in Section 2-(III) may be chosen depending only on $\psi_{0}, k, k_{1}$ and $k_{2}$, but independent of $w_{0}$.

PROof. Let $w_{c}(x, \phi)$ be the approximate solution mentioned in (III) with respect to the initial datum $w_{0}$. Then by the Oleinik's theory [9] it is suffcient to prove the following: For the constants $a, \delta, A_{0}, \cdots$ and $A_{5}$ which depend only on $\psi_{0}, k, k_{1}$ and $k_{2}$, there exists a sequence $\left\{\varepsilon_{n}\right\}$ depending on $w_{0}(\phi)$ and tending to zero as $n \rightarrow \infty$ such that for any $n$

$$
\begin{aligned}
w_{\iota_{n}}(x, \phi) & \geq V(x, \phi) \\
& \equiv w_{\iota_{n}}(x, 0)+f(\phi)\left(1+e^{-a x}\right) \text { in } G_{A_{0}}^{c_{n}} .
\end{aligned}
$$

Here $f(\psi)=A_{1} \psi^{4 / 3}+A_{2} \psi$ for $\psi \leq \delta, \quad A_{3} \geq f(\psi) \geq f(\delta),\left|f_{\psi}(\psi)\right| \leq A_{4}$ and $\left|f_{\psi \psi}(\psi)\right| \leq$ $A_{5}$ for $\phi \geq \delta$.

To show this, let $B_{0}, B_{1}$ and $B_{2}$ be sufficiently small constants such that

$$
B_{2}>B_{0} \text {, }
$$

$$
\frac{1}{2} k_{1} \exp \left\{-3 k_{2} X_{1} / k_{1}\right\} \geq B_{2} \text {. }
$$

Moreover we take sufficiently small constants $\delta, A_{1}, A_{2}$ and $A_{3}$ as follows :

$$
\delta<\psi_{0},
$$

$$
\begin{aligned}
& \min \left(B_{2}-B_{0}, k \delta\right) \geq 2 A_{3}>4\left(A_{1} \delta^{1 / 3}+A_{2}\right) \delta>0, \\
& \nu \frac{4}{9} \sqrt{A_{2}} A_{1} \geq \delta^{1 / 6}\left(k_{2}+B_{1}\right) .
\end{aligned}
$$

Then from (3.23) we may determine the desired monotone nondecreasing function $f \in C^{2}([0, \infty))$, constants $A_{4}$ and $A_{5}$.

Now let $w_{0} \in W$. From the condition

$$
w_{0}(\psi) \uparrow U^{2}(0) \text { as } \phi \uparrow \infty,
$$


we find a sequence $\left\{\psi_{n}\right\}$ such that $w_{0_{\varphi \varphi}}\left(\psi_{n}\right) \rightarrow 0$ as $n \rightarrow \infty$. Therefore we have

$$
\mu\left(\psi_{n}\right) x \geq-3 k_{2} X_{1}
$$

for any sufficiently large $n$ and for $x \in\left[0, X_{1}\right]$. Accordingly by (3.22) we can find a sequence $\left\{\varepsilon_{n}\right\}$ as follows :

$$
\varepsilon_{n}+\delta<\psi_{0},
$$

for $x \in\left[0, X_{1}\right]$

$$
\begin{aligned}
& \left(w_{0} \exp \left\{\mu x / w_{0}\right\}\right)\left(\varepsilon_{n}\right) \leq B_{0}, \\
& \left(\mu \exp \left\{\mu x / w_{0}\right\}\right)\left(\varepsilon_{n}\right) \leq B_{1}, \\
& \left(w_{0} \exp \left\{\mu x / w_{0}\right\}\right)\left(\varepsilon_{n}+\frac{1}{\varepsilon_{n}}\right) \geq B_{2} .
\end{aligned}
$$

Because $w_{0}\left(\varepsilon_{n}\right), \mu\left(\varepsilon_{n}\right) \downarrow 0$ as $\varepsilon_{n} \downarrow 0$ and $\mu\left(\varepsilon_{n}\right) / w_{0}\left(\varepsilon_{n}\right)$ is bounded by (2.6).

Now we can show the validity of (3.21) on the boundary of $G_{X_{1}}^{s_{n}}$ except the line $x=X_{1}$. On the line $x=0,(3.23)$ implies that for $\phi \leq \delta$

$$
\begin{gathered}
w_{0}\left(\varepsilon_{n}+\phi\right)-w_{0}\left(\varepsilon_{n}\right)=\int_{0}^{1} r w_{0}^{\prime}\left(\varepsilon_{n}+\theta \psi\right) d \theta \cdot \psi \geq k \psi \\
\geq 2\left(A_{1} \delta^{1 / 3}+A_{2}\right) \psi \geq 2\left(A_{1} \psi^{4 / 3}+A_{2} \psi\right) .
\end{gathered}
$$

For $\phi \geq \delta, \quad w_{0}\left(\varepsilon_{n}+\phi\right)-w_{0}\left(\varepsilon_{n}\right) \geq w_{0}\left(\varepsilon_{n}+\delta\right)-w_{0}\left(\varepsilon_{n}\right)=\delta \int_{0}^{1} w_{0}^{\prime}\left(\varepsilon_{n}+\theta \delta\right) d \theta \geq 2 A_{3} \geq$ $2 f\left(\varepsilon_{n}+\phi\right)$ by the definition of $f$ and also by (2.23).

Furthermore on the line $\phi=1 / \varepsilon_{n},(3.23)$ and (3.25) imply that

$$
\begin{aligned}
& \left(w_{0} \exp \left\{\mu x / w_{0}\right\}\right)\left(\varepsilon_{n}+\frac{1}{\varepsilon_{n}}\right)-\left(w_{0} \exp \left\{\mu x / w_{0}\right\}\right)\left(\varepsilon_{n}\right) \\
& \geq B_{2}-B_{0} \geq 2 A_{3} \text { for } x \in\left[0, X_{1}\right] .
\end{aligned}
$$

Since (3.21) is trivial on the line $\phi=0$, we obtain the assertion above mentioned.

Finally we consider

$$
L(V)=\nu \sqrt{V} f^{\prime \prime}(\phi)\left(1+e^{-a x}\right)-w_{\varepsilon}(x, 0)_{x}+a f(\psi) e^{-a x} .
$$

For $\phi \leq \delta$, from (3.24) and (3.25) it follows that

$$
\begin{aligned}
L(V) . & \geq \nu \frac{4}{9} \sqrt{A_{2}} A_{1} \delta^{-1 / 6}-\sup _{0 \leq x \leq X_{1}}\left(\mu \exp \left\{\mu x / w_{0}\right\}\right)\left(\varepsilon_{n}\right) \\
& \geq k_{2} \geq 2 p_{x}(x) .
\end{aligned}
$$

By the monotoneity of $f$ we can find a constant $d$ such that $f(\phi) \geq d>0$ for $\phi \geq \delta$. Thus there exist constants $A_{0}$ and a which satisfy 


$$
\begin{aligned}
& 0<A_{0}<X_{1}, a A_{0}=1, \\
& a d e^{-a A_{0}} \geq k_{2}+\nu \sqrt{k_{1}} A_{5}\left(1+e^{-a A_{0}}\right)+B_{1} .
\end{aligned}
$$

Then we obtain that $L(V) \geq 2 p_{x}(x)$ for $x \in\left[0, A_{0}\right]$ and for $\psi \geq \delta$.

Thus we conclude that $L\left(w_{s_{n}}\right)-L(V) \leq 0$ in $G_{A_{0}}^{s_{n}}$ and by the maximum principle (3.21) is valid.

q.e.d.

\section{Proofs of Theorems}

(I) First, we shall prove Theorem 1. Let $w(x, \phi)$ be Oleinik's local solution in $G_{A_{0}}$. From Corollary of Lemma 1 and Lemma 2 the section $w\left(A_{0}, \cdot\right)$ belongs to $\mathrm{I}_{M}{ }^{2+\alpha}$. Hence, from the facts (II) and (III) in Section 2, there exists a continued solution of Oleinik's local solution (By $w(x, \phi)$ we also denote this continuation). Furthermore by the same arguments as Lemma 1 this continuation belongs the class $P_{M}{ }^{2+\alpha}([0, A])$ for some $A>A_{0}$, and its section $w(x, \cdot)$ belongs to $I_{M}^{2+\alpha}$ for any $x(0 \leq x \leq A)$.

Let $s=\sup \left\{A\right.$; the above continuation exists in $\left.G_{A}\right\}$. Then we obtain the continued solution $w(x, \phi)$ belonging to $P_{M}{ }^{2+\alpha}([0, s))$. Furthermore, from Colorllary of Lemma 4, an inequality $s<X_{0}$ holds.

Assume that the point $(s, 0)$ is not the separation point. That is, for a sequence $A_{n}<s$ with $A_{n} \rightarrow s$ (as $n \rightarrow \infty$ ) there exist positive constants $m_{0}$, $\phi_{0}$ and a natural number $n_{0}$ which do not depend on $n$ and satisfy the following :

$$
\inf \left\{w_{\psi}\left(A_{n}, \psi\right) ; 0 \leq \psi \leq \psi_{0}\right\} \geq m_{0} \text { for } n \geq n_{0} .
$$

For an arbitrary but fixed $n \geq n_{0}$, if we consider the line $x=A_{n}$ as an initial position and the section $w\left(A_{n}, \cdot\right)$ as an initial datum, then there exists the solution of the problem (2.3), (2.4) and (2.5) in the domain $G_{A_{n}+B}$ for some constant $B$, which belongs to the class $P_{M^{2+\alpha}}\left(\left[A_{n}, A_{n}+B\right]\right)$. On the other hand from (4.1) and Lemma 5 the constant $B$ does not depend on $n \geq n_{0}$. Hence, for sufficiently large $n_{1}$ an inequality $B>s-A_{n_{1}}$ holds. Thus we get a solution of the problem (2.3), (2.4) and (2.5) in $G_{A_{n_{1}+B}}$ as a continuation of the solution $w(x, \phi) \in P_{M}^{2+\alpha}([0, s))$, which is contrary to the definition of $s$. This proves Theorem 1 .

We note that, by the same way as above, if $U(x)$ does not vanish and if there exists no separation point, then Oleinik's local solution can be continued to the infinity.

(II) To prove Theorem 2, we show a relation between the solution obtained in Theorem 1 and the Blasius solution, which is given as follows (see [3]). 
Let $f=f(\eta)$ be the solution of the problem :

$$
f^{\prime \prime \prime}+f f^{\prime \prime}=0 \text { in }[0, \infty),
$$

with conditions

$$
f(0)=f^{\prime}(0)=0 \quad \text { and } \quad f^{\prime}(\eta) \rightarrow 1 \quad \text { as } \quad \eta \rightarrow \infty .
$$

If we put

$$
\begin{aligned}
\eta & =\left(\frac{U\left(x_{0}\right)+c}{2 \nu x}\right)^{1 / 2} y, \\
u(x, y) & =\left(U\left(x_{0}\right)+c\right) f^{\prime}(\eta), \\
v(x, y) & =\nu^{1 / 2}\left(\frac{U\left(x_{0}\right)+c}{2 x}\right)^{1 / 2}\left(\eta f^{\prime}(\eta)-f(\eta)\right)
\end{aligned}
$$

for an arbitrary but fixed positive constant $c$ and the point $x_{0}$ given in Lemma 3 , then the function $(u, v)$ satisfies

$$
u u_{x}+v u_{y}=\nu u_{y y} \text { and } u_{x}+v_{y}=0 \text { in }(0, \infty) \times(0, \infty)
$$

with conditions

$$
u=v=0 \text { for } y=0 \text { and } x>0 .
$$

Using the transformation (2.2) and putting $w_{B}(x, \phi)=u^{2}(x, y)$, by definitions we obtain

$$
\begin{aligned}
& L\left(w_{B}\right)=0 \text { in }(0, \infty) \times(0, \infty), \\
& w_{B}(x, 0)=0 \text { for } x>0, \\
& w_{B}(x, \phi) \rightarrow\left(U\left(x_{0}\right)+c\right)^{2} \text { as } \phi \rightarrow \infty \text { pointwise in } x>0, \\
& w_{B}(x, \phi) \rightarrow\left(U\left(x_{0}\right)+c\right)^{2} \text { as } x \rightarrow 0 \text { pointwise in } \phi>0 .
\end{aligned}
$$

Now let $w(x, \phi)$ be the solution in Theorem 1 with $S\left(w_{0}\right)>x_{0}$. Then we obtain

$$
w(x, \phi) \leq w_{B}\left(x-x_{0}, \phi\right) \quad \text { in } \quad\left[x_{0}, S\left(w_{0}\right)\right) \times[0, \infty),
$$

where the point $x_{0}$ is given in Lemma 3.

In fact from (1.5) and Lemma 3 we have

$$
p_{x}(x) \geq 0 \text { for } x_{0} \leq x \leq X_{0} .
$$

Furthermore from Theorem 8.1 in [3] it follows that

$$
f^{\prime \prime}(\eta)>0 \text { for } \eta>0 \text {. }
$$

Hence, from (2.2), (4.2) and (4.7), we have 


$$
w_{B_{\psi}}(x, \phi)=\frac{2\left(U\left(x_{0}\right)+c\right)^{3 / 2}}{(2 \nu x)^{1 / 2}} f^{\prime \prime}(\eta)>0,
$$

since $\frac{\partial}{\partial \phi}=2 \frac{\partial}{\partial y}$ is valid.

Therefore from (4.4) and (4.8) we see that for a fixed positive number $\delta$ there exists a positive constant $\varepsilon_{0}$ such that

$$
w_{B}(\varepsilon, \phi)-w\left(x_{0}, \phi\right) \geq 0 \text { for } \delta \leq \phi<\infty \text { and } 0<\varepsilon<\varepsilon_{0} .
$$

Furthermore from (4.8) it follows that

$$
\begin{aligned}
& w_{B}(\varepsilon, \phi)-w\left(x_{0}, \phi\right) \\
& \quad=\phi \int_{0}^{1}\left(\frac{\partial w_{B}}{\partial \phi}(\varepsilon, t \psi)-\frac{\partial w}{\partial \phi}\left(x_{0}, t \psi\right)\right) d t \geq 0 \text { for } 0<\phi \leq \delta,
\end{aligned}
$$

provided $\varepsilon \ll 1$.

Hence we have

$$
w_{B}(\varepsilon, \phi)-w\left(x_{0}, \phi\right) \geq 0 \text { for } 0 \leq \phi<\infty .
$$

Moreover from (4.3), (4.4) and (4.6) we deduce that

$$
\begin{aligned}
& w_{B}\left(x-x_{0}+\varepsilon, 0\right)-w(x, 0)=0 \text { for } \quad x_{0} \leq x<S\left(w_{0}\right), \\
& L\left(w_{B}\left(x-x_{0}+\varepsilon, \phi\right)\right)-L(w) \leq 0 \text { in }\left[x_{0}, S\left(w_{0}\right)\right) \times[0, \infty) .
\end{aligned}
$$

Thus by the maximum principle we conclude that for any sufficiently small $\varepsilon>0$

$$
w(x, \phi) \leq w_{B}\left(x-x_{0}+\varepsilon, \phi\right) \quad \text { in } \quad\left[x_{0}, S\left(w_{0}\right)\right) \times[0, \infty),
$$

which implies (4.5).

(III) We shall prove Theorem 2. Let $x=x_{0}$ be given in Lemma 3. As in the proof of Corollary of Lemma 4, we may consider the line $x=x_{0}+\varepsilon$ as an initial position for sufficiently small $\varepsilon>0$. Furthermore from (4.5) we obtain

$$
w\left(x_{0}+\varepsilon, \phi\right) \leq w_{B}(\varepsilon, \phi) \leq k \psi \int_{0}^{1} p_{x}(t) d t
$$

for $0 \leq \psi \leq 2 / k$, if the constant $k$ is sufficiently large. Then the constant $k$ in (3.13) can be chosen independently of the section $w\left(x_{0}+\varepsilon, \cdot\right)$ for any solution with $S\left(w_{0}\right)>x_{0}$. Hence after a certain coordinates transformation the constant $A$ in $(3.19)$ can be also chosen independently of $w\left(x_{0}+\varepsilon, \cdot\right)$. Then from (3.20) the inequality (1.7) holds. The first part of Theorem 2 is proved. 
Finally we shall prove the second assertion of Theorem 2 . We define $w_{0}^{(\nu)} \in I_{M}{ }^{2+\alpha}(\nu, U)$ as follows :

$$
w_{0}^{(\nu)}=m \psi \chi(\psi)+B_{1} \psi^{3 / 2} \chi\left(\nu^{-5 / 2} \psi\right)-B_{2} \psi^{2} \chi\left(\nu^{-5 / 2} \psi\right)+(1-\chi(\psi)) U^{2}(0),
$$

where $B_{1}, B_{2}$ and $\chi$ are defined in Section 2-(II).

By simple calculations, we have

$$
w_{0}^{(\nu)} \rightarrow m \psi \chi(\phi)+(1-\chi(\psi)) U^{2}(0) \quad \text { in } \quad B^{1}([0, \infty)),
$$

$\nu \sqrt{w_{0}^{(\nu)}} \frac{d^{2} w_{0}^{(\nu)}}{d \psi^{2}} \rightarrow 0$ in $B^{0}\left(\left[\psi_{0}, \infty\right)\right)$ for any $\phi_{0}>0$ as $\nu \rightarrow 0,\left\{\nu \sqrt{w_{0}^{(\nu)}} \frac{d^{2} w_{0}^{(\nu)}}{d \psi^{2}}\right\}$ is contained in a bounded subset of $B^{0}([0, \infty))$.

Let $u_{0}^{(\nu)}(y)$ be the function $\in I^{2+\alpha}(\nu, U)$ which corresponds to $w_{0}^{(\nu)}(\phi)$ by the Von Mises' transformation restricted on $x=0$. i. e.,

$$
u_{0}\left(\int_{0}^{\varphi} \frac{d t}{\sqrt{w_{0}(t)}}\right)=\sqrt{w_{0}(\phi)}
$$

Then we obtain $u_{0}^{(\nu)} \rightarrow u_{0}$ in $B^{1}([0, \infty))$ as $\nu \rightarrow 0$ for some $u_{0}$. On the other hand, since $\left\{w_{0}^{(\nu)}\right\}$ is convergent in $B^{1}([0, \infty))$, the constant $k$ in $(3.13)$ with respect to $w_{0}^{(\nu)}$ is bounded and the sequence of the numbers $D$ in (3.19) tends to 1 as $\nu \rightarrow 0$. Hence we have $S\left(w_{0}^{(\nu)}\right) \rightarrow 0$ as $\nu \rightarrow 0$ by (3.20). This proves the second assertion of Theorem 2 .

\section{References}

[1] FIFE, P. C.: Considerations regarding the mathematical basis for Prandtl's boundary layer theory, Arch. Rat. Mech. Anal., 28, 184-216 (1968).

[2] GöRtLer, H.: A new series of calculation of steady laminar boundary layer flows, Jour. Math. Mech., 6, 1-66 (1957).

[3] Hartman, P.: Ordinary Differential Equations, John Wiley and Sons Inc., New York (1964).

[4] Horwarth, L.: Laminar boundary layers, Encyclopedia of Physics VIII/1 Fluid Dynamics I, ed. S. Fluge, Springer-Verlag, Berlin, 264-277 (1959).

[5] Il'in, A. M., Kalashnikov, A. S. and Oleinik, O. A.: Linear equations of the second order of parabolic type, Russian Math. Survey, 17-3, 3-146 (1962).

[ 6 ] Landau, L. D. and Lifshitz, E. M.: Fluid Mechanics, Pergamon Press, Oxford, 145-156 (1966).

[7] LIU, C. S. and LEE, C. H.: On the solution of the Prandtl boundary layer equations containing the point of zero skin friction, Arch. Rat. Mech. Anal., 79, 291-304 (1982).

[8] NiCKel, K.: Parabolic equations with applications to boundary layer theory, 
P. D. E. and Conti. Mech., ed. R. Langer, The Univ. Wisconsin Press, Madison, Wisconsin, 319-330 (1961).

[9] OlEINIK, O. A.: On a system of equations in boundary layer theory, U.S.S.R. Comp. Math. Phys., 3, 650-673 (1963).

[10] OlEiNiK, O. A.: Lecture N. 1-Lecture N. 5, Semi. Ist Naz. Alta Math., 1, 332436 (1965).

[11] Oleinik, O. A.: Weak solutions in the Sobolev sense for a system of boundary layer equations, Amer. Math. Soc. Transl. (2), 105, 247-264 (1976).

[12] Schlichting, H.: Boundary-Layer Theory, McGraw Hill Co., New York (1979).

[13] TANI, I.: On the solution of the laminar boundary layer equations, Jour. Phys. Japan 4, 149-154 (1949).

Department of Mathematics

Faculty of Science

Hokkaido University

Sapporo 060 Japan 